\title{
Research Article \\ Solving the Linear 1D Thermoelasticity Equations with Pure Delay
}

\author{
Denys Ya. Khusainov ${ }^{1}$ and Michael Pokojovy ${ }^{2}$ \\ ${ }^{1}$ Department of Cybernetics, Kyiv National Taras Shevchenko University, 64 Volodymyrska Street, Kyiv 01601, Ukraine \\ ${ }^{2}$ Department of Mathematics and Statistics, University of Konstanz, Universitätsstraße 10, 78457 Konstanz, Germany \\ Correspondence should be addressed to Michael Pokojovy; michael.pokojovy@uni-konstanz.de
}

Received 27 October 2014; Accepted 3 January 2015

Academic Editor: Harvinder S. Sidhu

Copyright ( 2015 D. Ya. Khusainov and M. Pokojovy. This is an open access article distributed under the Creative Commons Attribution License, which permits unrestricted use, distribution, and reproduction in any medium, provided the original work is properly cited.

\begin{abstract}
We propose a system of partial differential equations with a single constant delay $\tau>0$ describing the behavior of a one-dimensional thermoelastic solid occupying a bounded interval of $\mathbb{R}^{1}$. For an initial-boundary value problem associated with this system, we prove a well-posedness result in a certain topology under appropriate regularity conditions on the data. Further, we show the solution of our delayed model to converge to the solution of the classical equations of thermoelasticity as $\tau \rightarrow 0$. Finally, we deduce an explicit solution representation for the delay problem.
\end{abstract}

\section{Introduction}

Over the past half-century, the equations of thermoelasticity have drawn a lot of attention from the side of both mathematical and physical communities. Starting with the late 50 s and early 60 s of the last century, the necessity of a rational physical description for elastic deformations of solid bodies accompanied by thermal stresses motivated the more prominent mathematicians, physicists, and engineers to focus on this problem (see, e.g., $[1,2])$. As a consequence, many theories emerged, mainly in the cross-section of (nonlinear) field theory and thermodynamics, making it possible for the equations of thermoelasticity to be interpreted as an anelastic modification of the equations of elasticity (cf. [3] and the references therein). Both linear and nonlinear models and solution theories were proposed.

An initial-boundary value problem for the general linear equations of classical thermoelasticity in a bounded smooth domain $\Omega \subset \mathbb{R}^{n}$

$$
\begin{aligned}
& \rho \partial_{t t} u_{i}=\left(C_{i j k l} u_{k, l}\right)_{, j}-\left(m_{i j} T\right)_{, j}+\rho f_{i} \quad \text { in } \Omega \times(0, \infty), \\
& \rho c_{D} \partial_{t} T+m_{i j} \theta_{0} \partial_{t} u_{i, j}=\left(K_{i j} T_{, j}\right)_{, i}+\rho c_{D} r \quad \text { in } \Omega \times(0, \infty)
\end{aligned}
$$

was studied by Dafermos in [4]. Here, $\left[u_{i}\right]$ and $T$ denote the (unknown) displacement vector field and the absolute temperature, respectively. Further, $\rho>0$ is the material density, $\theta_{0}$ is a reference temperature rendering the body free of thermal stresses, $c_{D}$ is the specific heat capacity, $\left[C_{i j k l}\right]$ stands for Hooke's tensor, $\left[m_{i j}\right]$ is the stress-temperature tensor, $\left[K_{j}\right]$ is the heat conductivity tensor, $\left[f_{i}\right]$ represents the specific external body force, and $r$ is the external heat supply. Under usual initial conditions, appropriate normalization conditions to rule out the rigid motion as a trivial solution, and general boundary conditions

$$
\begin{gathered}
u_{i}=0 \quad \text { in } \Gamma_{1} \times(0, \infty), \\
\left(C_{i j k l} u_{k, l}-m_{i j} T\right) n_{j}+A_{i j} u_{j}=0 \quad \text { in } \operatorname{int}\left(\Gamma_{1}^{c}\right) \times(0, \infty), \\
T=0 \quad \text { in } \Gamma_{2} \times(0, \infty), \\
\left(K_{i j} T_{, j}\right) n_{i}+B T=0 \quad \text { in } \operatorname{int}\left(\Gamma_{2}^{c}\right) \times(0, \infty),
\end{gathered}
$$

where $\Gamma_{1}, \Gamma_{2} \subset \partial \Omega$ are relatively open, $\left[A_{i j}\right]$ denotes the "elasticity" modulus, and $B$ is heat transfer coefficient, Dafermos proved the global existence and uniqueness of finite energy solutions and studied their regularity as well as asymptotics 
as $t \rightarrow \infty$. In $1 \mathrm{D}$, even an exponential stability result for (1)(2) under all "reasonable" boundary conditions was shown by Hansen in [5].

In his work [6], Slemrod studied the nonlinear equations of $1 \mathrm{D}$ thermoelasticity in the Lagrangian coordinates

$$
\begin{aligned}
& \partial_{t t} u= \widehat{\psi}_{F F}\left(\partial_{x} u+1, \theta+T_{0}\right) \partial_{x x} u \\
&+\widehat{\psi}_{F T}\left(\partial u_{x}+1, \theta+T_{0}\right) \partial_{x} \theta \quad \text { in }(0,1) \times(0, \infty) \\
& \rho\left(\theta+T_{0}\right)\left(\widehat{\psi}_{T T}\left(\partial_{x} u+1, \theta+T_{0}\right) \partial_{t} \theta\right. \\
&\left.\quad+\widehat{\psi}_{F T}\left(\partial_{x} u+1, \theta+T_{0}\right) \partial_{x t} u\right) \\
&=\widehat{q}^{\prime}\left(\partial_{x} \theta\right) \partial_{x x} \theta \quad \text { in }(0,1) \times(0, \infty)
\end{aligned}
$$

for the unknown functions $u$ denoting the displacement of the rod and $\theta$ being a temperature difference to a reference temperature $T_{0}$ rendering the body free of thermal stresses. The functions $\widehat{\psi}$ and $\widehat{q}$ denote the Helmholtz free energy and the heat flux, respectively, and are assumed to be given. Finally, $\rho>0$ is the material density in the references configuration. Under appropriate boundary conditions (when the boundary is free of tractions and is held at a constant temperature or when the body is rigidly clamped and thermally insulated) as well as usual initial conditions for both unknown functions, a local existence theorem for (4) was proved by additionally imposing a regularity and compatibility condition. For sufficiently small initial data, the local classical solution could be globally continued. At the same time, when studying (4) in the whole space, large data are known to lead to a blow-up in final time (cf. [7]).

Racke and Shibata studied in [8] (4) under homogeneous Dirichlet boundary conditions for both $u$ and $\theta$. Under appropriate smoothness assumptions, they proved the global existence and exponential stability for the classical solutions to the problem. In contrast to Slemrod [6], their method was using spectral analysis rather than ad hoc energy estimates obtained by differentiating the equations with respect to $t$ and $x$. A detailed overview of further recent developments in the field of classical thermoelasticity and corresponding references can be found in the monograph [9] by Jiang and Racke.

The classical equations of thermoelasticity outlined above, being a hyperbolic-parabolic system, provide a rather good macroscopic description in many real-world applications. At the same time, they sometimes fail when being used to model thermoelastic stresses in some other situations, in particular, in extremely small bodies exposed to heat pulses of large amplitude (see, e.g., [10]) and so forth. To address these issues, a new theory, commonly referred to as the theory of hyperbolic thermoelasticity or second sound thermoelasticity, has emerged. In contrast to the classical thermoelasticity, parabolic equation (2) is replaced with a hyperbolic first-order system

$$
\begin{gathered}
\rho c_{D} \partial_{t} T+m_{i j} \theta_{0} \partial_{t} u_{i, j}=q_{i, i}+\rho c_{D} r \quad \text { in } \Omega \times(0, \infty), \\
\tau_{i j} \partial_{t} q_{i}+q_{i}+K_{i j} T_{, j}=0 \quad \text { in } \Omega \times(0, \infty)
\end{gathered}
$$

with $\left[q_{i}\right]$ and $\left[\tau_{i j}\right]$ denoting the heat flux and the relaxation tensor, respectively. Both linear and nonlinear versions of the equations of hyperbolic thermoelasticity (1), (5)-(6) have been studied in the literature. See, for example, [11] by Messaoudi and Said-Houari for a proof of global wellposedness of the 1D system in the whole space or Irmscher's work [12] for the global well-posedness of nonlinear problem for rotationally symmetric data in a bounded rotationally symmetric domain of $\mathbb{R}^{3}$. In a bounded $1 \mathrm{D}$ domain, a quantitative stability comparison between the classical and the hyperbolic system was presented by Irmscher and Racke in [13]. For a detailed overview on hyperbolic thermoelasticity, we refer the reader to [14] by Chandrasekharaiah and [15] by Racke.

A unified approach establishing a connection between the classical and hyperbolic thermoelasticity was developed by Tzou in [16, 17]. Namely, he proposed to view (6) with $\tau_{i j} \equiv \tau$ as a first-order Taylor approximation of the equation

$$
q_{i}(\mathbf{x}, t+\tau)+K_{i j} T_{, j}(\mathbf{x}, t)=0 \quad \text { for }(\mathbf{x}, t) \in \Omega \times(-\tau, \infty)
$$

being equivalent to the delay equation

$$
q_{i}(\mathbf{x}, t)+K_{i j} T_{, j}(\mathbf{x}, t-\tau)=0 \quad \text { for }(\mathbf{x}, t) \in \Omega \times(0, \infty) .
$$

More generally, higher-order Taylor expansion to the dual phase lag constitutive equation

$$
\begin{aligned}
& q_{i}\left(\mathbf{x}, t+\tau_{1}\right)+K_{i j} T_{, j}\left(\mathbf{x}, t+\tau_{2}\right) \\
& \quad=0 \text { for }(\mathbf{x}, t) \in \Omega \times\left(-\max \left\{\tau_{1}, \tau_{2}\right\}, \infty\right)
\end{aligned}
$$

can be considered. Together with (1)-(2), (5), this leads to the so-called dual phase lag thermoelasticity studied by Racke (cf. references in [15, page 415]).

If no Taylor expansion with respect to $\tau$ is carried out in (8), it can be shown that the corresponding system is illposed when being considered in the same topology as the original system of classical thermoelasticity (cf. [18]); that is, the system is lacking a continuous dependence of solution on the data. Moreover, the delay law (8) can, in general, contradict the second law of thermodynamics as shown in [19].

Nonetheless, it remains desirable to understand the dynamics of equations of thermoelasticity originated from delayed material laws. One of the first attempts to obtain a well-posedness result for a partial differential equation with pure delay is due to Rodrigues et al. In their paper [20], Rodrigues et al. studied a heat equation with pure delay in an appropriate Frechét space and showed the delayed Laplacian to generate a $C_{0}$-semigroup on this space. Further, they investigated the spectrum of the infinitesimal generator. Though their approach can essentially be carried over to the equations of thermoelasticity with pure delay derived in Section 2, we propose a new approach in this paper preserving the Hilbert space structure of the space and thus the connection to the classical equations of thermoelasticity. To the authors' best knowledge, no results on thermoelasticity with delay in the highest order terms have been previously published in the literature. At the same time, we refer the 
reader to the works by Khusainov et al. [21-24], in which the authors studied the well-posedness and controllability for the heat and/or the wave equation on a finite time horizon. In their recent paper [25], Khusainov et al. exploited the $L^{2}$-maximum regularity theory to prove a global wellposedness and asymptotic stability results for a regularized heat equation with delay.

The present paper has the following outline. In Section 2, we give a physical model for linear thermoelasticity based on delayed material laws. For the sake of simplicity, we present a $1 \mathrm{D}$ model though our approach can easily be carried over to the general multidimensional case. Next, in Section 3, we prove the well-posedness of this model in an appropriate Hilbert space framework and discuss the small parameter asymptotics, that is, the behavior of solutions as $\tau \rightarrow 0$. Further, in Section 4, we deduce an explicit solution representation formula. Finally, in the Appendix, we summarize some seminal results on the delayed exponential function and Cauchy problems with pure delay.

\section{Model Description}

We consider a solid body occupying an axis-aligned rectangular domain of $\mathbb{R}^{3}$. Assuming that the body motion is purely longitudinal with respect to the first space variable $x$ (cf. [6, page 100]), deformation gradient, stress and strain tensors, and so forth are diagonal matrices and a complete rational description of the original $3 \mathrm{D}$ body motion can be reduced to studying the $1 \mathrm{D}$ projection $\Omega=(0, l), l>0$, of the body onto the $x$-axis as displayed in Figure 1. Hence, in the following, we restrict ourselves to considering the relevant physical values only in $x$-direction.

Let the functions $u: \bar{\Omega} \times[0, \infty) \rightarrow \mathbb{R}$ and $\theta:$ $\bar{\Omega} \times[0, \infty) \rightarrow \mathbb{R}$ denote the body displacement and its relative temperature measured with respect to a reference temperature $\theta_{0}>0$ rendering the body free of thermal stresses, respectively. We restrict ourselves to the Lagrangian coordinates and write $\sigma, \varepsilon, S, q: \bar{\Omega} \times[0, \infty) \rightarrow \mathbb{R}$ for the stress field, strain field, entropy field, or the heat flux, respectively. With $\rho>0$ denoting the material density, the momentum conservation law as well as the linearized entropy balance law reads as

$$
\begin{array}{cc}
\rho \partial_{t t} u(x, t)+\partial_{x} \sigma(x, t)=\rho r(x, t) & \text { for } x \in \Omega, t>0, \\
\theta_{0} \partial_{t} S(x, t)+\partial_{x} q(x, t)=h(x, t) & \text { for } x \in \Omega, t>0,
\end{array}
$$

where $r: \bar{\Omega} \times[0, \infty) \rightarrow \mathbb{R}$ and $h: \bar{\Omega} \times[0, \infty) \rightarrow \mathbb{R}$ are a known volume force acting on the body and an internal heat source.

Assuming physical linearity for the strain field, the strain can be decomposed into elastic strain $\varepsilon^{e}$ and thermal stress $\varepsilon^{t}$. Further, assuming $\left|\theta(t, x) / \theta_{0}\right| \ll 1$ uniformly with respect to $x \in \bar{\Omega}, t \geq 0$, we can postulate

$$
\varepsilon^{t}(x, t)=\alpha \theta(x, t) \quad \text { for } x \in \Omega, t>0,
$$

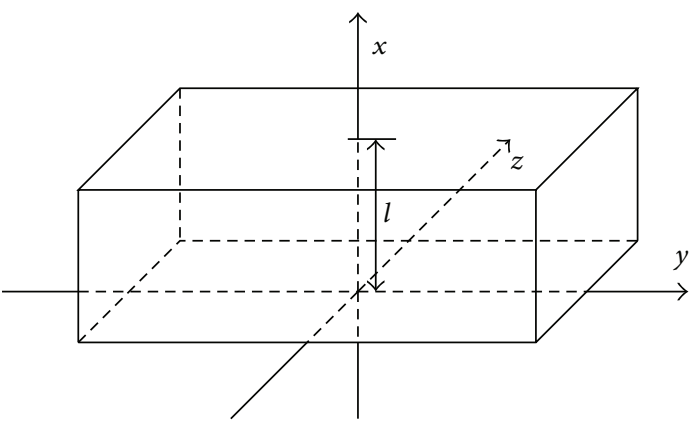

FIGURE 1: 3D rectangular solid body.

where $\alpha>0$ denotes the thermal expansion coefficient. Exploiting the second law of thermodynamics for irreversible processes, we obtain (cf. [3, page 3])

$$
S(x, t)=\alpha B \varepsilon^{e}(x, t)+\frac{\rho c_{\rho}}{\theta_{0}} \theta(x, t) \quad \text { for } x \in \Omega, t>0
$$

with $c_{\rho}>0$ standing for the specific heat capacity and $B \in \mathbb{R}$ denoting the bulk modulus.

In our further considerations, we depart from the classical material laws and use their delay counterparts. Let $\tau>0$ be a positive time delay. In the sequel, all functions are supposed to be defined on $\bar{\Omega} \times[-\tau, \infty)$. Assuming a delay feedback between the stress and the strain as well as the heat flux and the temperature gradient, Hooke's law with pure delay reads as (cf. [1])

$$
\begin{array}{r}
\sigma(x, t)=\left(B+\frac{4}{3} G\right) \varepsilon^{e}(x, t-\tau)+B \varepsilon^{t}(x, t-\tau) \\
\text { for } x \in \Omega, \quad t>0
\end{array}
$$

with $G>0$ denoting the shear modulus. Similarly, we consider a delay version of Fourier's law given as

$$
q(x, t)=-\kappa \partial_{x} \theta(x, t-\tau) \quad \text { for } x \in \Omega, t>0,
$$

where $\kappa>0$ stands for the thermal conductivity. Assuming the elastic strain tensor to be equal to the displacement gradient, we have

$$
\varepsilon^{e}(x, t)=\partial_{x} u(x, t) \quad \text { for } x \in \Omega, t>0 .
$$

We further postulate the following relation:

$$
\partial_{t} \partial_{x} u(x, t)=\partial_{x} \partial_{t} u(x, t-\tau) \quad \text { for } x \in \Omega, t>0
$$

which is a delayed counterpart of Schwarz's theorem. Finally, we also modify (12) to introduce a delay feedback between the entropy, the elastic strain tensor, and the temperature

$$
S(x, t)=\alpha B \varepsilon^{e}(x, t-\tau)+\frac{\rho c_{\rho}}{\theta_{0}} \theta(x, t-\tau)
$$

$$
\text { for } x \in \Omega, \quad t>0 \text {. }
$$


Exploiting now (10), (13)-(17), we obtain

$$
\begin{gathered}
\rho \partial_{t t} u(x, t)-\left(B+\frac{4}{3} G\right) \partial_{x x} u(x, t-\tau)+\alpha B \partial_{x} \theta(x, t-\tau) \\
=f(x, t) \quad \text { for } x \in \Omega, t>0 \\
\rho c_{\rho} \partial_{t} \theta(x, t)-\kappa \partial_{x x} \theta(x, t-\tau)+\alpha \theta_{0} B \partial_{t x} u(x, t-\tau) \\
=h(x, t) \quad \text { for } x \in \Omega, t>0 \\
\partial_{t} \partial_{x} u(x, t)-\partial_{x} \partial_{t} u(x, t-\tau)=0 \text { for } x \in \Omega, t>0 .
\end{gathered}
$$

To close (18), appropriate boundary and initial conditions for $u$ and $\theta$ are required. In the following, we prescribe homogeneous Dirichlet boundary conditions for $u$ and homogeneous Neumann boundary conditions for $\theta$ given as

$$
\begin{array}{r}
u(0, t)=u(l, t)=0, \\
\partial_{x} \theta(0, t)=\partial_{x} \theta(l, t)=0 \\
\text { for } t>0 .
\end{array}
$$

This particular choice of boundary conditions not only turns out to be convenient for our further mathematical considerations but also is a physically relevant one. Similar to the thermoelasticity with second sound, it is one of the combinations typically arising when studying micro- and nanoscopic strings or plates (cf. [13]).

The initial conditions are given over the whole history period $(\tau, 0)$ and read as

$$
\begin{array}{r}
u(x, 0)=u^{0}(x), \quad u(x, t)=u_{\tau}^{0}(x, t) \\
\text { for } x \in \Omega, \quad t \in(-\tau, 0), \\
\partial_{t} u(x, 0)=u^{1}(x), \quad \partial_{t} u(x, t)=u_{\tau}^{1}(x, t) \\
\text { for } x \in \Omega, \quad t \in(-\tau, 0), \\
\theta(x, 0)=\theta^{0}(x), \quad \theta(x, t)=\theta_{\tau}^{0}(x, t) \\
\text { for } x \in \Omega, \quad t \in(-\tau, 0)
\end{array}
$$

with known $u^{0}, u^{1}, \theta^{0}: \Omega \rightarrow \mathbb{R}$ and $u_{\tau}^{0}, u_{\tau}^{1}, \theta_{\tau}^{0}: \Omega \times(-\tau, 0) \rightarrow$ $\mathbb{R}$.

\section{Well-Posedness and Limit $\tau \rightarrow 0$}

Letting $a:=(B+(4 / 3) G) / \rho, b:=\alpha B / \rho, c:=\kappa / \rho c_{\rho}, d:=\alpha \theta_{0} B /$ $\rho c_{\rho}$ and $f(x, t):=r(x, t)$, and $g(x, t):=\left(1 / \rho c_{\rho}\right) h(x, t)$ for $x \in \bar{\Omega}, t \geq 0$, (18) can be rewritten as

$$
\begin{gathered}
\partial_{t t} u(x, t)-a \partial_{x x} u(x, t-\tau)+b \partial_{x} \theta(x, t-\tau) \\
=f(x, t) \quad \text { for } x \in \Omega, t>0, \\
\partial_{t} \theta(x, t)-c \partial_{x x} \theta(x, t-\tau)+d \partial_{t x} u(x, t-\tau) \\
=g(x, t) \quad \text { for } x \in \Omega, t>0, \\
\partial_{x} u(x, t)-\partial_{x} u(x, t-\tau)=0 \text { for } x \in \Omega, t>0
\end{gathered}
$$

subject to the boundary conditions from (19) and initial conditions from (20). Introducing a new vector of unknown functions,

$$
\begin{array}{r}
\mathbf{V}(x, t)=\left(\begin{array}{c}
V^{1}(x, t) \\
V^{2}(x, t) \\
V^{3}(x, t)
\end{array}\right):=\left(\begin{array}{c}
\partial_{t} u(x, t) \\
\partial_{x} u(x, t) \\
\theta(x, t)
\end{array}\right) \\
\text { for } x \in \bar{\Omega}, \quad t \in[-\tau, T] .
\end{array}
$$

Equations (21) can be transformed to

$$
\partial_{t} \mathbf{V}(x, t)+\mathbf{B V}(x, t-\tau)=\mathbf{F}(x, t) \quad \text { for } x \in \Omega, t \in(0, T)
$$

with the differential matrix operator and the right-hand side

$$
\begin{array}{rr}
\mathbf{B}:=\left(\begin{array}{ccc}
0 & -a \partial_{x} & b \partial_{x} \\
-\partial_{x} & 0 & 0 \\
d \partial_{x} & 0 & -c \partial_{x x}
\end{array}\right), \quad \mathbf{F}(x, t):=\left(\begin{array}{c}
f(x, t) \\
0 \\
g(x, t)
\end{array}\right) \\
& \text { for } x \in \bar{\Omega}, \quad t>0,
\end{array}
$$

respectively.

Exploiting (19) and the definition of $V$, the boundary conditions for $V$ read as

$$
\begin{array}{r}
V^{1}(0, t)=V^{1}(l, t)=0, \quad \partial_{x} V^{3}(0, t)=\partial_{x} V^{3}(l, t)=0 \\
\text { for } t>0,
\end{array}
$$

whereas the initial conditions are given by

$$
\begin{array}{r}
\mathbf{V}(x, 0)=\mathbf{V}^{0}(x), \\
\mathbf{V}(x, t)=\mathbf{V}_{\tau}^{0}(x, t) \\
\text { for } x \in \Omega, \quad t \in(-\tau, 0)
\end{array}
$$

with

$$
\mathbf{V}^{0}(x)=\left(\begin{array}{c}
u^{0} \\
u^{1} \\
\theta^{0}
\end{array}\right), \quad \mathbf{V}_{\tau}^{0}(x, t)=\left(\begin{array}{c}
u_{\tau}^{1}(x, t) \\
\partial_{x} u_{\tau}^{0}(x, t) \\
\theta_{\tau}^{0}(x, t)
\end{array}\right)
$$

for $x \in \bar{\Omega}, \quad t \in[-\tau, 0]$.

Note that (18)-(20), (23), (25), and (26) are equivalent for; if the vector $V$ is known, $u$ and $\theta$ are uniquely determined by

$$
\begin{aligned}
& u(x, t)= \begin{cases}u^{0}(x)+\int_{0}^{t} V^{1}(x, s) \mathrm{d} s, & \text { for } t \geq 0, \\
u_{\tau}^{0}(x, t), & \text { for } t \in[-\tau, 0),\end{cases} \\
& \theta(t, x)= \begin{cases}V^{3}(x, t), & \text { for } t \geq 0, \\
\theta_{\tau}^{0}(x, t), & \text { for } t \in[-\tau, 0) .\end{cases}
\end{aligned}
$$


Therefore, in the sequel, we consider the following equivalent first-order-in-time problem:

$$
\begin{array}{r}
V^{1}(0, t)=V^{1}(l, t)=0, \\
\partial_{x} V^{3}(0, t)=\partial_{x} V^{3}(l, t)=0 \\
\text { for } t>0, \\
\mathbf{V}(x, 0)=\mathbf{V}^{0}(x), \\
\mathbf{V}(x, t)=\mathbf{V}_{\tau}^{0}(x, t) \\
\text { for } x \in \Omega, \quad t \in(-\tau, 0) .
\end{array}
$$$$
\partial_{t} \mathbf{V}(x, t)+\mathbf{B V}(x, t-\tau)=\mathbf{F}(x, t) \quad \text { for } x \in \Omega, t>0,
$$

For our well-posedness investigations, we need a solution notion for (29). To this end, appropriate functional spaces have to be introduced. We start with the "naïve" approach by using the case $\tau=0$ as a reference situation. We introduce the Hilbert space $X:=L^{2}(\Omega) \times L^{2}(\Omega) \times L^{2}(\Omega)$ equipped with the dot product

$$
\begin{aligned}
\langle\mathbf{V}, \mathbf{W}\rangle_{X}:= & \left\langle V^{1}, W^{1}\right\rangle_{L^{2}(\Omega)}+a\left\langle V^{2}, W^{2}\right\rangle_{L^{2}(\Omega)} \\
& +\frac{b}{d}\left\langle V^{3}, W^{3}\right\rangle_{L^{2}(\Omega)} \quad \text { for } \mathbf{V}, \mathbf{W} \in X
\end{aligned}
$$

and define the operator

$$
\mathscr{B}: D(\mathscr{B}) \subset X \longrightarrow X, \quad V \longmapsto \mathbf{B} V
$$

with the domain

$$
D(\mathscr{B}):=\left\{\mathbf{V} \in H_{0}^{1}(\Omega) \times H^{1}(\Omega) \times H^{2}(\Omega)\left|\partial_{x} V^{3}\right|_{\partial \Omega}=0\right\} .
$$

See [26, Section 3] for the definition of Sobolev spaces. With this notation, (29) can be written in the equivalent form

$$
\begin{gathered}
\partial_{t} \mathbf{V}(x, t)+\mathscr{B} \mathbf{V}(x, t-\tau)=\mathbf{F}(x, t) \quad \text { for } x \in \Omega, t>0, \\
\mathbf{V}(x, 0)=\mathbf{V}^{0}(x), \\
\mathbf{V}(x, t)=\mathbf{V}_{\tau}^{0}(x, t)
\end{gathered}
$$$$
\text { for } x \in \Omega, \quad t \in(-\tau, 0) \text {. }
$$

Under a classical solution to (33) on $[-\tau, T]$ for any $T>0$, one would naturally understand a function $\mathbf{V} \in C^{0}([-\tau, T]$, $D(\mathscr{B})) \cap C^{1}([0, T], X)$ satisfying the equations pointwise.

We know from [5] that the linear operator $\mathscr{B}$ is accretive and satisfies $D(\mathscr{B})=D\left(\mathscr{B}^{*}\right)$. Its spectrum $\sigma(\mathscr{B})$ only consists of isolated eigenvalues $\lambda_{n} \in \mathbb{C}, n \in \mathbb{N}_{0}$, of finite multiplicity with $\operatorname{Re} \lambda_{n} \geq 0, n \in \mathbb{N}$, and $\lambda_{n} \rightarrow \infty$ as $n \rightarrow \infty$. The corresponding eigenfunctions $\left(\Psi_{n}\right)_{n \in \mathbb{N}} \subset D(\mathscr{B})$ build an orthonormal basis of $X$. Unfortunately, from [18, Theorem 1.1] we know that (33) is ill-posed in $X$. Hence, a different solution notion should be adopted. As we already mentioned in Section 1, we want to preserve the Hilbert space structure of the problem and thus cannot follow the approach developed by Rodrigues et al. in [20].

For $T>0$, we define the space $X_{T}:=\left\{V \in \bigcap_{k=0}^{\infty} D\left(\mathscr{B}^{k}\right) \mid\right.$ $\left.\|V\|_{X_{T}}<\infty\right\}$ equipped with the scalar product induced by the norm

$$
\begin{aligned}
\|\mathbf{V}\|_{X_{T}}: & =\left\|e^{T|\mathscr{B}|} \mathbf{V}\right\|_{D\left(\mathscr{B}^{2}\right)} \\
= & \left(\sum_{n=0}^{\infty}\left(1+\left|\lambda_{n}\right|^{2}+\left|\lambda_{n}\right|^{4}\right)\right. \\
& \left.\cdot \exp \left(2 T\left|\lambda_{n}\right|\right)\left|\left\langle\mathbf{V}, \Psi_{n}\right\rangle_{X}\right|^{2}\right)^{1 / 2}
\end{aligned}
$$

$$
\text { for } \mathbf{V} \in X_{T} \text {. }
$$

Hence, $X_{T}$ is closed subspace of $X$ and thus a Hilbert space. Moreover, $X_{T}$ is dense in $X$ since $\left(\Phi_{n}\right)_{n} \subset X_{T}$. Indeed, for $n \in \mathbb{N}$, we have $\Psi_{n} \in \bigcap_{k=0}^{\infty} D\left(\mathscr{B}^{k}\right)$ and $\left\|\Psi_{n}\right\|_{X_{T}}^{2}=\left(1+\left|\lambda_{n}\right|^{2}+\right.$ $\left.\left|\lambda_{n}\right|^{4}\right) \exp \left(2 T\left|\lambda_{n}\right|\right)<\infty$.

Restricting $\mathscr{B}$ to the closed subspace $X_{T}$ of $X$, we trivially obtain a bounded linear operator $\mathscr{B}_{T}: X_{T} \rightarrow D\left(\mathscr{B}^{2}\right)$ since for $\mathbf{V} \in X_{T}$

$$
\begin{gathered}
\left\|\mathscr{B}_{T} \mathbf{V}\right\|_{D\left(\mathscr{B}^{2}\right)}^{2}=\left\|\mathscr{B}_{T} \mathbf{V}\right\|_{X}^{2}+\left\|\mathscr{B}_{T} \mathbf{V}\right\|_{X}^{2}+\left\|\mathscr{B}^{2} \mathscr{B}_{T} \mathbf{V}\right\|_{X}^{2} \\
=\sum_{n=1}^{\infty}\left(1+\left|\lambda_{n}\right|^{2}+\left|\lambda_{n}\right|^{4}\right)\left|\lambda_{n}\right|^{2}\left|\left\langle\mathbf{V}, \Psi_{n}\right\rangle_{X}\right|^{2} \\
\leq \frac{1}{2 T^{2}} \sum_{n=1}^{\infty}\left(1+\left|\lambda_{n}\right|^{2}+\left|\lambda_{n}\right|^{4}\right)\left|\exp \left(2 T\left|\lambda_{n}\right|\right)\right| \\
\cdot\left|\left\langle\mathbf{V}, \Psi_{n}\right\rangle_{X}\right|^{2} \leq \frac{1}{2 T^{2}}\|\mathbf{V}\|_{X_{T}}^{2} .
\end{gathered}
$$

Now, restricting (33) to $X_{T}$, we obtain

$$
\begin{array}{r}
\partial_{t} \mathbf{V}(x, t)+\mathscr{B}_{T} \mathbf{V}(x, t-\tau)=\mathbf{F}(x, t) \\
\text { for } x \in \Omega, \quad t \in(0, T), \\
\mathbf{V}(x, 0)=\mathbf{V}^{0}(x), \\
\mathbf{V}(x, t)=\mathbf{V}_{\tau}^{0}(x, t) \\
\text { for } x \in \Omega, \quad t \in(-\tau, 0) .
\end{array}
$$

Following the approach in [21], we introduce for $t \in \mathbb{R}$ the delayed exponential function

$$
\exp _{\tau}\left(\mathscr{B}_{T}, t\right):= \begin{cases}0_{L(X)}, & t<-\tau, \\ \operatorname{id}_{X}+\sum_{k=1}^{\lfloor t / \tau\rfloor+1} \frac{(t-(k-1) \tau)^{k}}{k !} \mathscr{B}_{T}^{k}, & t \geq-\tau .\end{cases}
$$



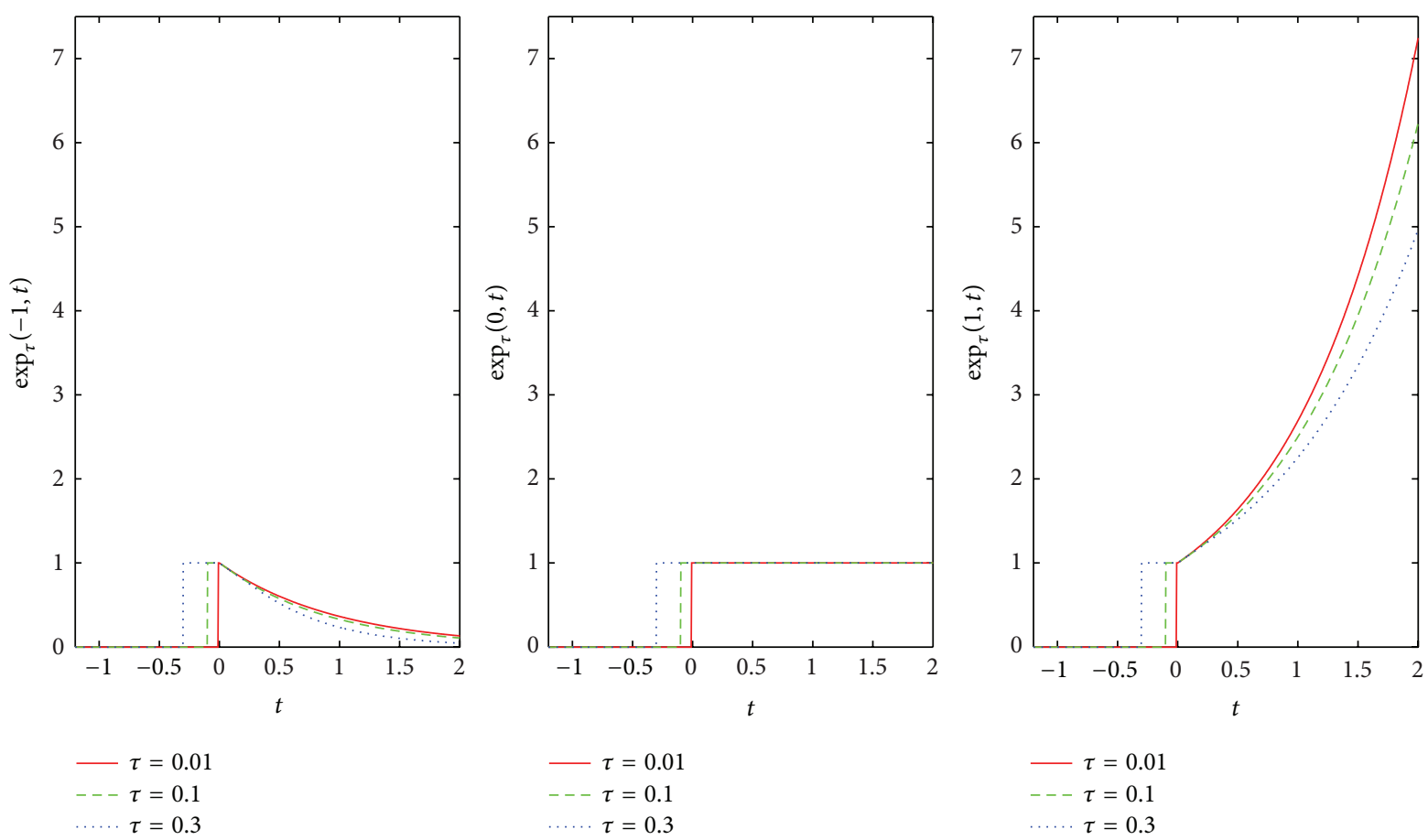

FIGURE 2: Delayed exponential function.

Figure 2 displays the delayed exponential function for the case where $\mathscr{B}_{T}$ is a real number.

Obviously, for any $t \in \mathbb{R}, \exp _{\tau}\left(-\mathscr{B}_{T}, t\right) \in \bigcap_{k=0}^{\infty} D\left(\mathscr{B}^{k}\right)$. Moreover, we have

$$
\left\|\exp _{\tau}\left(-\mathscr{B}_{T}, t\right)\right\|_{L\left(X_{T}, D\left(\mathscr{B}^{2}\right)\right)} \leq 1 \quad \text { for } t \in(-\infty, T]
$$

uniformly in $\tau>0$ since

$$
\begin{aligned}
&\left\|\exp _{\tau}\left(-\mathscr{B}_{T}, t\right) \mathbf{V}\right\|_{D\left(\mathscr{B}^{2}\right)}^{2} \\
&=\left\|\exp _{\tau}\left(-\mathscr{B}_{T}, t\right) \mathbf{V}\right\|_{X}^{2} \\
&+\left\|\mathscr{B}_{\exp _{\tau}}\left(-\mathscr{B}_{T}, t\right) \mathbf{V}\right\|_{X}^{2}+\left\|\mathscr{B}^{2} \exp _{\tau}\left(-\mathscr{B}_{T}, t\right) \mathbf{V}\right\|_{X}^{2} \\
&=\sum_{n=0}^{\infty}\left(1+\left|\lambda_{n}\right|^{2}+\left|\lambda_{n}\right|^{4}\right)\left|\exp _{\tau}\left(-\lambda_{n}, t\right)\right|^{2}\left|\left\langle\mathbf{V}, \Psi_{n}\right\rangle_{X}\right|^{2} \\
& \leq \sum_{n=0}^{\infty}\left(1+\left|\lambda_{n}\right|^{2}+\left|\lambda_{n}\right|^{4}\right) \\
& \cdot\left(1+\sum_{k=1} \frac{\mid t / \tau]+1}{(t-(k-1) \tau)^{k}}\left|\lambda_{n}\right|^{k}\right)^{2} \\
& \cdot\left|\left\langle\mathbf{V}, \Psi_{n}\right\rangle_{X}\right|^{2} \\
& \leq \sum_{n=0}^{\infty}\left(1+\left|\lambda_{n}\right|^{2}+\left|\lambda_{n}\right|^{4}\right) \exp \left(2 T\left|\lambda_{n}\right|\right) \\
& \cdot\left|\left\langle\mathbf{V}, \Psi_{n}\right\rangle_{X}\right|^{2}=\|\mathbf{V}\|_{X_{T}}^{2} \quad \text { for } \mathbf{V} \in X_{T} .
\end{aligned}
$$

Here, $L(X, Y)$ denotes the space of bounded, linear operators from $X$ to $Y$ equipped with the standard operator topology.

Now, we can prove the following well-posedness result.

Theorem 1. For $T>0$, let $\mathbf{V}^{0} \in X_{T}, \mathbf{V}_{\tau}^{0} \in C^{0}\left([-\tau, 0], X_{T}\right)$ with $\mathbf{V}_{\tau}^{0}(\cdot, 0)=\mathbf{V}^{0}$ and let $\mathbf{F} \in C^{0}\left([0, T], X_{T}\right)$. Then (36) possess a unique classical solution $\mathbf{V} \in C^{0}([-\tau, T], D(\mathscr{B})) \cap$ $C^{1}([0, T], X)$ explicitly given by

$$
\begin{aligned}
& \mathbf{V}(\cdot, t) \\
& = \begin{cases}\mathbf{V}_{\tau}^{0}(\cdot, t), & t \in[-\tau, 0), \\
\mathbf{V}^{0}, & t=0, \\
\exp _{\tau}\left(-\mathscr{B}_{T}, t-\tau\right) \mathbf{V}^{0} & \\
-\mathscr{B}_{T} \int_{-\tau}^{0} \exp _{\tau}\left(-\mathscr{B}_{T}, t-2 \tau-s\right) & \\
& \cdot \mathbf{V}_{\tau}^{0}(s) d s \\
\quad+\int_{0}^{t} \exp _{\tau}\left(-\mathscr{B}_{T}, t-\tau-s\right) \mathbf{F}(\cdot, s) d s, & t \in(0, T] .\end{cases}
\end{aligned}
$$

Proof. First, we prove uniqueness of classical solutions. Assuming $\mathbf{V}$ and $\mathbf{W}$ to be classical solutions of (36), we conclude that their difference $\mathbf{Z}:=\mathbf{V}-\mathbf{W} \in C^{0}([-\tau, T], D(\mathscr{B})) \cap$ $C^{1}([0, T], X)$ is a classical solution to

$$
\begin{gathered}
\partial_{t} \mathbf{Z}(x, t)+\mathscr{B}_{T} \mathbf{Z}(x, t-\tau)=\mathbf{0} \quad \text { for } x \in \Omega, t \in(0, T), \\
\mathbf{Z}(x, 0)=\mathbf{0}, \quad \mathbf{Z}(x, t)=\mathbf{0} \quad \text { for } x \in \Omega, t \in(-\tau, 0) .
\end{gathered}
$$


Multiplying these equations with $\Psi_{n}$ in the inner product of $X$, we further deduce that $\mathbf{Z}_{n}(t, \cdot):=\left\langle\mathbf{Z}(t, \cdot), \Psi_{n}\right\rangle_{X}$, $t \in[-\tau, T]$, is a classical solution to the scalar delay differential equation

$$
\begin{gathered}
\dot{\mathbf{Z}}_{n}(t)+\lambda_{n} \mathbf{Z}_{n}(t-\tau)=\mathbf{0} \text { for } t \in(0, T), \\
\mathbf{Z}_{n}(0)=\mathbf{0}, \quad \mathbf{Z}_{n}(t)=\mathbf{0} \text { for } x \in \Omega, t \in(-\tau, 0) .
\end{gathered}
$$

From Theorem A.4 in the Appendix, the later equations are known to be uniquely solvable by $\mathbf{Z}_{n} \equiv \mathbf{0}$. Hence, $\mathbf{Z}_{n} \equiv \mathbf{0}$ for all $n \in \mathbb{N}$. With $\left(\Psi_{n}\right)_{n \in \mathbb{N}}$ being a basis of $X$, this implies $\mathbf{Z} \equiv \mathbf{0}$, and, therefore, $\mathbf{V} \equiv \mathbf{W}$.

For the existence proof, we show that the function V in (40) is a classical solution to (36). Performing the diagonalization, we obtain for $t \in[0, T]$

$$
\begin{aligned}
\mathbf{V}(\cdot, t) & \\
= & \sum_{n=1}^{\infty} \exp _{\tau}\left(-\lambda_{n}, t-\tau\right)\left\langle\mathbf{V}^{0}, \Psi_{n}\right\rangle_{X} \Psi_{n} \\
& -\sum_{n=1}^{\infty} \lambda_{n}\left(\int_{-\tau}^{0} \exp _{\tau}\left(-\lambda_{n}, t-2 \tau-s\right)\left\langle\mathbf{V}_{\tau}^{0}(s), \Psi_{n}\right\rangle_{X} \mathrm{~d} s\right) \Psi_{n} \\
& +\sum_{n=1}^{\infty}\left(\int_{0}^{t} \exp _{\tau}\left(-\lambda_{n}, t-\tau-s\right)\left\langle\mathbf{F}(\cdot, s), \Psi_{n}\right\rangle_{X} \mathrm{~d} s\right) \Psi_{n} \\
\equiv & \sum_{n=1}^{\infty} \mathbf{V}_{n}(t) \Psi_{n} .
\end{aligned}
$$

From [25], we know $\mathbf{V}_{n} \in C^{0}([-\tau, T], \mathbb{C}) \cap C^{1}([0, T], \mathbb{C})$ for all $n \in \mathbb{N}$. Further, by the virtue of (38), the series converges uniformly in $D(\mathscr{B})$ and its derivative converges uniformly in $X$. Hence, the limiting function lies in $C([-\tau, T], D(\mathscr{B})) \cap$ $C^{1}([0, T], X)$ and thus possesses the regularity of a classical solution. Finally, using the properties of scalar delay exponential (cf. [25]), we easily verify that $\mathbf{V}$ solves (36).

Taking into account inequality (38) and applying Hölder's inequality to (40), we obtain the following estimate.

Corollary 2. The solution $\mathbf{V}$ continuously depends on the data in sense of the estimate

$$
\begin{aligned}
\|\mathbf{V}\|_{C^{0}([0, T], X)} \leq & \left\|\mathbf{V}^{0}\right\|_{X_{T}}+\tau\left\|\mathbf{V}_{\tau}^{0}\right\|_{C^{0}\left([0, T], X_{T}\right)} \\
& +\sqrt{T}\|\mathbf{F}\|_{L^{2}\left(0, T ; X_{T}\right)} \quad \text { for } T>0 .
\end{aligned}
$$

For the rest of this section, we want to study the behavior of system (33) as $\tau \rightarrow 0$. Let $\tau_{0}>0$ and $T>0$ be fixed. Similar to $X_{T}$, we consider the Hilbert space $Y_{T}:=\{V \in$ $\left.\bigcap_{k=0}^{\infty} D\left(\mathscr{B}^{k}\right) \mid\|V\|_{Y_{T}}<\infty\right\}$ equipped with the scalar product induced by the norm

$$
\begin{aligned}
\|\mathbf{V}\|_{Y_{T}}:=\left(\sum_{n=0}^{\infty}\left(1+\left|\lambda_{n}\right|^{2}\right)\right. & \\
\cdot & \exp \left(2\left(1+\left|\lambda_{n}\right| \exp \left(\tau_{0}\left|\lambda_{n}\right|\right)\right) T \lambda_{n}\right) \\
& \left.\cdot\left|\left\langle\mathbf{V}, \Psi_{n}\right\rangle_{X}\right|^{2}\right)^{1 / 2} \text { for } \mathbf{V} \in Y_{T} .
\end{aligned}
$$

Obviously, $Y_{T} \hookrightarrow X_{T}$. For simplicity, despite a slight abuse of notation, we let $\mathscr{B}_{T}$ now denote the part of $\mathscr{B}$ in $Y_{T}$ (namely, [27, page 139]). Formally, the limiting system of (33) as $\tau \rightarrow 0$ is given by

$$
\begin{gathered}
\partial_{t} \overline{\mathbf{V}}(x, t)+\mathscr{B}_{T} \overline{\mathbf{V}}(x, t)=\mathbf{F}(x, t) \quad \text { for } x \in \Omega, t \in(0, T), \\
\overline{\mathbf{V}}(x, 0)=\mathbf{V}^{0}(x) \quad \text { for } x \in \Omega .
\end{gathered}
$$

From [27, Corollary 3.3.13], we know that $-\mathscr{B}_{T}$ generates a uniformly bounded $C_{0}$-semigroup $\left(\exp \left(-\mathscr{B}_{T} t\right)\right)_{t \geq 0}$ of bounded linear operators on $Y_{T}$. The unique classical solution to (46) can then be written using Duhamel's formula as

$$
\begin{aligned}
\overline{\mathbf{V}}(\cdot, t)= & \exp \left(-\mathscr{B}_{T} t\right) \mathbf{V}^{0} \\
& +\int_{0}^{t} \exp \left(-\mathscr{B}_{T}(t-s)\right) \mathbf{F}(s) \mathrm{d} s \quad \text { for } t \in[0, T] .
\end{aligned}
$$

Theorem A.3 from the Appendix implies the following.

Lemma 3. There holds

$$
\begin{array}{r}
\left\|\exp _{\tau}\left(-\mathscr{B}_{T}, t-\tau\right)-\exp \left(-\mathscr{B}_{T} t\right)\right\|_{L\left(Y_{T}, D(\mathscr{B})\right)} \leq \tau \\
\text { for } t \in[0, T] .
\end{array}
$$

Now, we can prove the following.

Theorem 4. Let $\mathbf{V}^{0} \in Y_{T}, \mathbf{F} \in C^{0}\left([0, \infty), Y_{T}\right)$ be fixed. For $\tau>0$, let $\mathbf{V}_{\tau}^{0} \in C^{0}\left([-\tau, 0], Y_{T}\right)$ with $\mathbf{V}_{\tau}^{0}(0)=\mathbf{V}^{0}$ and $\lim \sup _{\tau \rightarrow 0}\left\|\mathbf{V}_{\tau}^{0}\right\|_{L^{1}\left(0, \tau ; Y_{T}\right)}<\infty$. Denoting with $V(\cdot ; \tau)$ the classical solution of (36) corresponding to the initial data $\mathbf{V}^{0}$, $\mathbf{V}_{\tau}^{0}$ and the right-hand side $\mathbf{F}$, one has

$$
\|\mathbf{V}(\cdot ; \tau)-\overline{\mathbf{V}}(\cdot ; \tau)\|_{C^{0}([0, T], X)}=O(\tau) \quad \text { as } \tau \longrightarrow 0 .
$$

Proof. Using the representation formulas for $\mathbf{V}$ and $\overline{\mathbf{V}}$, we can estimate for any $t \in[0, T]$

$$
\begin{aligned}
& \|\mathbf{V}(\cdot ; \tau)-\overline{\mathbf{V}}(\cdot ; \tau)\|_{X} \\
& \leq\left\|\exp _{\tau}\left(-\mathscr{B}_{T}, t-\tau\right)-\exp \left(-\mathscr{B}_{T} t\right)\right\|_{L\left(Y_{T}, X\right)}\left\|\mathbf{V}^{0}\right\|_{Y_{T}} \\
& \quad+\int_{-\tau}^{0}\left\|\mathscr{B}_{T} \exp _{\tau}\left(-\mathscr{B}_{T}, t-2 \tau-s\right)\right\|_{L\left(Y_{T}, X\right)}\left\|\mathbf{V}_{\tau}^{0}(s)\right\|_{Y_{T}} \mathrm{~d} s \\
& \quad+\int_{0}^{t}\left\|\exp _{\tau}\left(-\mathscr{B}_{T}, t-\tau-s\right)-\exp \left(-\mathscr{B}_{T}(t-s)\right)\right\|_{L\left(Y_{T}, X\right)} \\
& \quad \cdot\|\mathbf{F}(\cdot, s)\|_{Y_{T}} \mathrm{~d} s \\
& \leq \tau\left\|\mathbf{V}^{0}\right\|_{Y_{T}}+\tau(1+\tau) \limsup _{\tau \rightarrow 0}\left\|\mathbf{V}_{\tau}^{0}\right\|_{L^{1}\left(-\tau, 0 ; Y_{T}\right)} \\
& \quad+\tau T\|\mathbf{F}\|_{L^{\infty}\left(0, T ; Y_{T}\right)}=O(\tau) \quad \text { as } \tau \longrightarrow 0 .
\end{aligned}
$$

This finishes the proof. 


\section{Explicit Solution Representation}

In this section, we want to deduce an explicit representation of solutions to (36) in the form of a Fourier series with respect to an orthogonal basis $\left(\Phi_{n}\right)_{n \in \mathbb{N}_{0}}$ of $X$ (and thus of $X_{T}$ ) given by

$$
\begin{gathered}
\Phi_{n}(x) \\
= \begin{cases}\sqrt{\frac{1}{2 l}}(0,1,1)^{T}, & \text { if } n=0, \\
\sqrt{\frac{2}{3 l}}\left(\sin \left(v_{n} x\right), \cos \left(v_{n} x\right), \cos \left(v_{n} x\right)\right)^{T}, & \text { otherwise }\end{cases}
\end{gathered}
$$$$
\text { for } x \in \bar{\Omega}, \quad n \in \mathbb{N}_{0}
$$

with

$$
v_{n}:=\frac{\pi n}{L} \quad \text { for } n \in \mathbb{N}_{0} .
$$

Note that the sequence $\left(\Phi_{n}\right)_{n \in \mathbb{N}_{0}}$ does not coincide, in general, with the eigenfunctions $\left(\Psi_{n}\right)_{n \in \mathbb{N}_{0}}$ but, at the same time, $\left(\Phi_{n}\right)_{n \in \mathbb{N}_{0}} \subset D\left(\mathscr{B}_{T}\right)$ constitutes a basis of $D\left(\mathscr{B}_{T}\right)$. To this end, we assume that the conditions of Theorem 1 are satisfied which yields a unique classical solution $\mathbf{V} \epsilon$ $C^{0}([-\tau, \infty), D(\mathscr{B})) \cap C^{1}([0, \infty), X)$.

Denoting $\Phi_{n}=\left(\Phi_{n}^{1}, \Phi_{n}^{2}, \Phi_{n}^{3}\right)^{T}$ and computing the componentwise Fourier coefficients

$$
\begin{aligned}
& V_{n}^{0, k}=\left\langle V^{0, k}, \Phi_{n}^{k}\right\rangle_{L^{2}(\Omega)}, \\
& V_{\tau, n}^{0, k}(t)=\left\langle V_{\tau}^{0, k}(\cdot, t), \Phi_{n}^{k}\right\rangle_{L^{2}(\Omega)} \quad \text { for } t \in[-\tau, 0], \\
& F_{n}^{k}(t)=\left\langle F^{k}(\cdot, t), \Phi_{n}^{k}\right\rangle_{L^{2}(\Omega)} \text { for } t \geq 0
\end{aligned}
$$

for $n \in \mathbb{N}_{0}$ and $k=1,2,3$, we get the following Fourier expansions:

$$
\begin{aligned}
& \mathbf{V}^{0}=\sum_{n=0}^{\infty}\left(V_{n}^{0,1} \Phi_{n}^{1}, V_{n}^{0,2} \Phi_{n}^{2}, V_{n}^{0,3} \Phi_{n}^{3}\right), \\
& \mathbf{V}_{\tau}^{0}(\cdot, t)=\sum_{n=0}^{\infty}\left(V_{\tau, n}^{0,1} \Phi_{n}^{1}, V_{n}^{0,2} \Phi_{n}^{2}, V_{n}^{0,3} \Phi_{n}^{3}\right) \quad \text { for } t \in[-\tau, 0] \\
& \mathbf{F}(\cdot, t)=\sum_{n=0}^{\infty}\left(F_{n}^{1} \Phi_{n}^{1}, F_{n}^{2} \Phi_{n}^{2}, F_{n}^{3} \Phi_{n}^{3}\right) \quad \text { for } t \geq 0
\end{aligned}
$$

uniformly in $\bar{\Omega}$ (due to the Sobolev embedding theorem). Similarly, the solution $\mathbf{V}$ can be expanded into Fourier series

$$
\mathbf{V}(\cdot, t)=\sum_{n=0}^{\infty}\left(V_{n}^{1}(t) \Phi_{n}^{1}, V_{n}^{2}(t) \Phi_{n}^{1}, V_{n}^{3}(t) \Phi_{n}^{1}\right)
$$

for some $V_{n, k} \in C^{0}([-\tau, \infty), \mathbb{C}) \cap C^{1}([0, \infty), \mathbb{C}), n \in \mathbb{N}_{0}, k=$ $1,2,3$, to be determined later. Using this ansatz and letting

$$
\mathbf{B}_{n}:=\left(\begin{array}{ccc}
0 & a v_{n} & -b v_{n} \\
-v_{n} & 0 & 0 \\
d v_{n} & 0 & c v_{n}^{2}
\end{array}\right)
$$

we observe that (36) decompose into a sequence of ordinary delay differential equations

$$
\begin{gathered}
\dot{\mathbf{V}}_{n}(t)=-\mathbf{B}_{n} \mathbf{V}_{n}(t-\tau)+\mathbf{F}_{n}(t) \quad \text { for } t>0, \\
\mathbf{V}_{n}(0)=\mathbf{V}_{n}^{0}, \quad \mathbf{V}_{n}(t)=\mathbf{V}_{\tau, n}^{0}(t) \quad \text { for } t \in(-\tau, 0) .
\end{gathered}
$$

By the virtue of Theorem A.4, for any $n \in \mathbb{N}_{0}$, the unique solution to (57) is given by

$$
\begin{aligned}
& \mathbf{V}_{n}(t)
\end{aligned}
$$

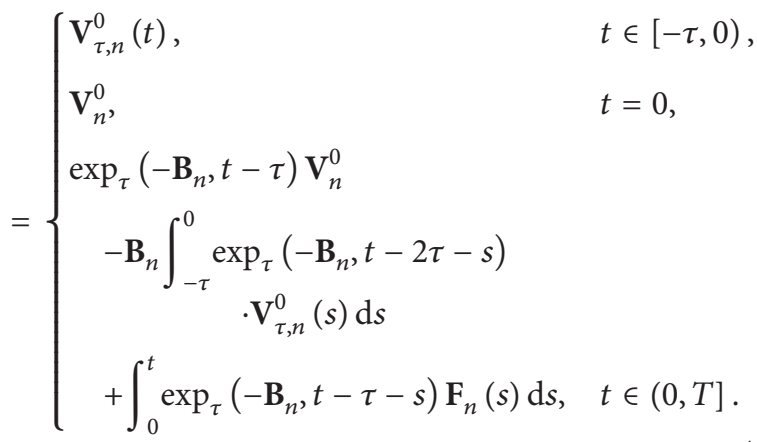

To explicitly compute the function given in (58), we need to diagonalize the matrix $\mathbf{B}_{n}$.

Lemma 5. Let

$$
\begin{aligned}
& \Delta_{0}=c^{2} v_{n}^{4}-3(a+b d) v_{n}^{2}, \\
& \Delta_{1}=-2 c^{3} v_{n}^{6}+9 c(a+b d) v_{n}^{4}-27 a c v_{n}^{4}, \\
& C=\sqrt[3]{\frac{1}{2}\left(\Delta_{1}+\sqrt{\Delta_{1}^{2}-4 \Delta_{0}^{3}}\right)},
\end{aligned}
$$

where $\sqrt{\cdot}$ and $\sqrt[3]{\cdot}$ stand for the main branch of complex square and cubic roots. The spectrum of $\mathbf{B}_{n}$ consists of three eigenvalues

$$
\mu_{n, k}= \begin{cases}0, & n=0, \\ \frac{1}{3}\left(c \nu_{n}^{2}-C e^{2 i k \pi / 3}-e^{-2 i k \pi / 3} \frac{\Delta_{0}}{C}\right), & \text { otherwise }\end{cases}
$$

for $k=0,1,2$ with $i$ denoting the imaginary unit.

Proof. For $n=0$, we have $v_{n}=0$ and therefore $\mathbf{B}_{n}=$ $\mathbf{0}_{3 \times 3}$. Hence, 0 is the only eigenvalue of $\mathbf{B}_{n}$ with an algebraic multiplicity of 3 .

Now, let us assume $n>1$. To compute the eigenvalues of $\mathbf{B}_{n}$, we consider the characteristic polynomial

$$
\begin{aligned}
P_{n}(\mu):= & \operatorname{det}\left(\mathbf{B}_{n}-\mu \mathbf{I}_{3 \times 3}\right)=\mu^{3}-c \nu_{n}^{2} \mu^{2} \\
& +(a+b d) \nu_{n}^{2} \mu-a c \nu_{n}^{4} \text { for } \mu \in \mathbb{C} .
\end{aligned}
$$

Since the matrix

$$
\mathbf{B}_{n}:=\left(\begin{array}{ccc}
1 & 0 & 0 \\
0 & \frac{1}{a} & 0 \\
& a & b \\
0 & 0 & \frac{\bar{d}}{d}
\end{array}\right)\left(\begin{array}{ccc}
0 & a v_{n} & -b v_{n} \\
-a v_{n} & 0 & 0 \\
b v_{n} & 0 & \frac{c d}{b} v_{n}^{2}
\end{array}\right)
$$


has real components and is skew-symmetrizable, it has to possess one real and two complex-conjugate eigenvalues. Thus, introducing the expressions

$$
\begin{aligned}
& \Delta_{0}=c^{2} v_{n}^{4}-3(a+b d) v_{n}^{2}, \\
& \Delta_{1}=-2 c^{3} v_{n}^{6}+9 c(a+b d) v_{n}^{4}-27 a c v_{n}^{4}, \\
& C=\sqrt[3]{\frac{1}{2}\left(\Delta_{1}+\sqrt{\Delta_{1}^{2}-4 \Delta_{0}^{3}}\right)}
\end{aligned}
$$

we obtain the three roots $\mu_{n, 1}, \mu_{n, 2}, \mu_{n, 3}$ of $\widetilde{P}_{n}$ (cf. [28, page 179])

$$
\mu_{n, k}=\frac{1}{3}\left(c v_{n}^{2}-C e^{2 i k \pi / 3}-e^{-2 i k \pi / 3} \frac{\Delta_{0}}{C}\right),
$$

where $\sqrt{\cdot}$ and $\sqrt[3]{\cdot}$ stand for the main branch of complex square and cubic roots.

Lemma 6. Eigenvectors $v_{n, k}, k=0,1,2$, of $\mathbf{B}_{n}$ corresponding to the eigenvalues $\mu_{n, k}$ of $\mathbf{B}_{n}$ from Lemma 5 are given by

$$
\mathbf{v}_{n, k}= \begin{cases}\mathbf{e}_{k}, & \text { if } n=0 \\
\left(\begin{array}{c}
-b v_{n} \mu_{n, k} \\
b v_{n}^{2} \\
a v_{n}^{2}+\mu_{n, k}^{2}
\end{array}\right), & \text { otherwise }\end{cases}
$$

with $\mathbf{e}_{1}=(1,0,0)^{T}, \mathbf{e}_{2}=(0,1,0)^{T}$, and $\mathbf{e}_{3}=(0,0,1)^{T}$.

Proof. Since the first case $n=0$ is obvious, we only consider the case $n>1$. For $k \in\{0,1,2\}$, we consider the matrix

$$
\mu_{n, k} \mathbf{I}_{3 \times 3}-\mathbf{B}_{n}=\left(\begin{array}{ccc}
\mu_{n, k} & -a v_{n} & b v_{n} \\
\nu_{n} & \mu_{n, k} & 0 \\
-d \nu_{n} & 0 & \mu_{n, k}-c v_{n}^{2}
\end{array}\right) .
$$

The latter is singular since $\alpha_{n, k}$ is an eigenvalue of $\mathbf{B}_{n}$. Further, due to the fact that

$$
\operatorname{det}\left(\mathbf{B}_{n}\right)=a c v_{n}^{4}>0,
$$

$\mathbf{B}_{n}$ is invertible and, therefore, $\mu_{n, k} \neq 0$. We want to find a nontrivial vector $\mathbf{v}_{n, k} \in \mathbb{R}^{3}$ satisfying

$$
\left(\alpha_{n, k} \mathbf{I}_{3 \times 3}-\mathbf{B}_{n}\right) v_{n, k}=\mathbf{0}_{3 \times 1} \text {. }
$$

Thus, we can apply a Gauss-Jordan iteration to the former matrix and find

$$
\mu_{n, k} \mathbf{I}_{3 \times 3}-\mathbf{B}_{n} \sim\left(\begin{array}{ccc}
\mu_{n, k} & -a v_{n} & b v_{n} \\
0 & \mu_{n, k}^{2}-a v_{n}^{2} & -b v_{n}^{2} \\
0 & -a d v_{n}^{2} & \mu_{n, k}^{2}-c v_{n}^{2} \mu_{n, k}+b d v_{n}^{2}
\end{array}\right)
$$

Since the latter matrix must be singular, the third row must be proportional to the second one. Thus, $(68)$ is equivalent to

$$
\left(\begin{array}{ccc}
\mu_{n, k} & -a v_{n} & b v_{n} \\
0 & \mu_{n, k}^{2}+a v_{n}^{2} & -b v_{n}^{2}
\end{array}\right) \mathbf{v}_{n, k}=\mathbf{0}_{3 \times 1} .
$$

Since the rank of this matrix is 2 , the equation above yields only one eigenvector

$$
\mathbf{v}_{n, k}=\left(\begin{array}{c}
-b v_{n} \mu_{n, k} \\
b v_{n}^{2} \\
a v_{n}^{2}+\mu_{n, k}^{2}
\end{array}\right)
$$

being determined up to a multiplicative constant.

Note that $\mathbf{v}_{n, 1}, \mathbf{v}_{n, 2}$, and $\mathbf{v}_{n, 3}$ are linearly independent, but, in general, not orthonormal.

Letting now

$$
\mathbf{D}_{n}:=\operatorname{diag}\left(\mu_{n, 1}, \mu_{n, 2}, \mu_{n, 3}\right) \text {, }
$$

we obtain a singular value decomposition for $\mathbf{B}_{n}$

$$
\mathbf{B}_{n}=\mathbf{S}_{n} \mathbf{D}_{n} \mathbf{S}_{n}^{-1}
$$

with an invertible matrix

$$
\mathbf{S}_{n}=\left(\begin{array}{lll}
\mathbf{v}_{n, 1} & \mathbf{v}_{n, 2} & \mathbf{v}_{n, 3}
\end{array}\right)^{T} .
$$

Exploiting now Corollary A.2 from Appendix, (58) can finally be written as

$$
\begin{aligned}
& \mathbf{V}_{n}(t) \\
& = \begin{cases}\mathbf{V}_{\tau, n}^{0}(t), & t \in[-\tau, 0), \\
\mathbf{V}_{n}^{0}, & t=0, \\
\mathbf{S}_{n} \exp _{\tau}\left(-\mathbf{D}_{n}, t-\tau\right) \mathbf{S}_{n}^{-1} V_{n}^{0} & \\
-\mathbf{S}_{n} \mathbf{D}_{n} \int_{-\tau}^{0} \exp _{\tau}\left(-\mathbf{D}_{n}, t-2 \tau-s\right) & \\
\cdot \mathbf{S}_{n}^{-1} \mathbf{V}_{\tau, n}^{0}(s) \mathrm{d} s & t \in(0, T], \\
+\int_{0}^{t} \mathbf{S}_{n} \exp _{\tau}\left(-\mathbf{D}_{n}, t-\tau-s\right) & \\
\cdot \mathbf{S}_{n}^{-1} \mathbf{F}_{n}(s) \mathrm{d} s, & \end{cases}
\end{aligned}
$$

where the inverse of $\mathbf{S}_{n}$ is given by the Laplace formula$$
\mathbf{S}_{n}^{-1}
$$

$$
\begin{aligned}
&=\left(\begin{array}{ccc}
S_{n}^{22} S_{n}^{33}-S_{n}^{23} S_{n}^{32} & -S_{n}^{12} S_{n}^{33}+S_{n}^{13} S_{n}^{32} & S_{n}^{12} S_{n}^{23}-S_{n}^{13} S_{n}^{22} \\
-S_{n}^{21} S_{n}^{33}+S_{n}^{23} S_{n}^{31} & S_{n}^{11} S_{n}^{33}-S_{n}^{13} S_{n}^{31} & -S_{n}^{11} S_{n}^{23}+S_{n}^{13} S_{n}^{21} \\
S_{n}^{21} S_{n}^{32}-S_{n}^{22} S_{n}^{32} & -S_{n}^{11} S_{n}^{32}+S_{n}^{12} S_{n}^{31} & S_{n}^{11} S_{n}^{22}-S_{n}^{12} S_{n}^{21}
\end{array}\right) \\
& \cdot\left(S_{n}^{11} S_{n}^{22} S_{n}^{33}+S_{n}^{12} S_{n}^{23} S_{n}^{31}+S_{n}^{13} S_{n}^{21} S_{n}^{32}-S_{n}^{31} S_{n}^{22} S_{n}^{13}\right. \\
&\left.-S_{n}^{32} S_{n}^{23} S_{n}^{11}-S_{n}^{33} S_{n}^{21} S_{n}^{12}\right)^{-1} .
\end{aligned}
$$




\section{Appendix}

\section{Ordinary Delay Differential Equations}

Let $X$ be a (real or complex) Hilbert space and let $\tau>0$ be arbitrary. For a bounded linear operator $\mathscr{B}$ on $X$, we consider the following ordinary delay differential equation:

$$
\begin{aligned}
& \partial_{t} u(t)=\mathscr{B} u(t-\tau)+f(t) \quad \text { for } t>0, \\
& u(0)=u^{0}, \\
& u(t)=u_{\tau}^{0} \quad \text { for } t \in(-\tau, 0)
\end{aligned}
$$

for some $u^{0} \in X, u_{\tau}^{0} \in L^{2}(-\tau, 0 ; X)$, and $f \in L_{\text {loc }}^{2}(0, \infty ; X)$.

Since the delay exponential function

$$
\exp _{\tau}(\mathscr{B}, t)= \begin{cases}0_{L(X)}, & t<-\tau, \\ \operatorname{id}_{X}+\sum_{k=1}^{\lfloor t / \tau\rfloor+1} \frac{(t-(k-1) \tau)^{k}}{k !} \mathscr{B}^{k}, & t \geq-\tau\end{cases}
$$

is an operator polynomial in $\mathscr{B}$ piecewise with respect to $t$, we obviously have the following representation.

Theorem A.1. Let $\mathcal{S}: X \rightarrow X$ be an isomorphism. Then

$$
\exp _{\tau}(\mathscr{B}, t)=\mathcal{S} \exp _{\tau}\left(\mathcal{S}^{-1} \mathscr{B} \mathcal{S}, t\right) \mathcal{S}^{-1} \quad \text { for } t \in \mathbb{R}
$$

Corollary A.2. If $X \in\left\{\mathbb{R}^{d}, \mathbb{C}^{d}\right\}, d \in \mathbb{N}$, and $\mathscr{B}=\mathbf{B}$ is diagonalizable over $\mathbb{C}$, that is, if there exists a diagonal matrix $\mathbf{D}=\operatorname{diag}\left(\lambda_{1}, \ldots, \lambda_{n}\right), \lambda_{1}, \ldots, \lambda_{d} \in \mathbb{C}$, and an invertible $\mathbf{S} \in \mathbb{C}^{d \times d}$ such that $\mathbf{A}=\mathbf{S D S}^{-1}$, then

$$
\begin{aligned}
\exp _{\tau}(\mathbf{B}, t) & =\mathbf{S} \exp _{\tau}(\mathbf{D}, t) \mathbf{S}^{-1} \\
& =\mathbf{S} \operatorname{diag}\left(\exp _{\tau}\left(\lambda_{1}, t\right), \ldots, \exp _{\tau}\left(\lambda_{n}, t\right)\right) \mathbf{S}^{-1} \\
& \text { for } t \in \mathbb{R} .
\end{aligned}
$$

Theorem A.3. Let $b \in \mathbb{C}, T>0$, and $\tau_{0}>0$ and let $\alpha:=$ $1+|b| \exp \left(\tau_{0}|b|\right)$. Then, for any $\tau \in\left(0, \tau_{0}\right]$,

$$
\left|\exp _{\tau}(b, t-\tau)-\exp (b t)\right| \leq \tau \exp (\alpha T|b|) \quad \text { for } t \in[0, T] \text {. }
$$

Proof. Let $\tau \in\left(0, \tau_{0}\right]$. For $t \in[0, \tau]$, the claim immediately follows from the mean value theorem for integration. We use mathematical induction to prove for any $k \in \mathbb{N}$

$$
\begin{aligned}
\left|\exp _{\tau}(b, t-\tau)-\exp (b t)\right| & \leq \tau \exp (\alpha k \tau|b|) \\
\text { for } t & \in((k-1) \tau, k \tau] .
\end{aligned}
$$

Indeed, assuming that the claim is true for some $k \in \mathbb{N}$, the fundamental theorem of calculus yields for $t \in(k \tau,(k+1) \tau]$

$$
\begin{aligned}
& \left|\exp _{\tau}(b, t-\tau)-\exp (b t)\right| \\
& \leq \tau \exp (\alpha k \tau|b|) \\
& +\int_{k \tau}^{(k+1) \tau}\left|\frac{\mathrm{d}}{\mathrm{d} s} \exp _{\tau}(b, s-\tau)-\frac{\mathrm{d}}{\mathrm{d} s} \exp (b s)\right| \mathrm{d} s \\
& \leq \tau \exp (\alpha k \tau|b|) \\
& +|b| \int_{k \tau}^{(k+1) \tau}\left|\exp _{\tau}(b, s-2 \tau)-\exp (b s)\right| \mathrm{d} s \\
& \leq \tau \exp (\alpha k \tau|b|) \\
& +|b| \int_{k \tau}^{(k+1) \tau}\left|\exp _{\tau}(b, s-2 \tau)-\exp (b(s-\tau))\right| \mathrm{d} s \\
& +|b| \int_{k \tau}^{(k+1) \tau}|\exp (b s)-\exp (b(s-\tau))| \mathrm{d} s \\
& \leq \tau \exp (\alpha k \tau|b|) \\
& +|b| \int_{(k-1) \tau}^{k \tau}\left|\exp _{\tau}(b, s-\tau)-\exp (b s)\right| \mathrm{d} s \\
& +|b| \int_{k \tau}^{(k+1) \tau} \int_{s-\tau}^{s}\left|\frac{\mathrm{d}}{\mathrm{d} \sigma} \exp (b \sigma)\right| \mathrm{d} \sigma \mathrm{d} s \\
& \leq \tau \exp (\alpha k \tau|b|)+\tau^{2}|b| \exp (\alpha k \tau|b|) \\
& +\tau^{2}|b|^{2} \exp ((k+1) \tau|b|) \\
& \leq \tau \exp (\alpha k \tau|b|)\left(1+\tau|b|+\tau|b|^{2} \exp (\tau|b|)\right) \\
& \leq \tau \exp (\alpha k \tau|b|)(1+\tau|b|(1+\tau|b| \exp (\tau|b|))) \\
& \leq \tau \exp (\alpha k \tau|b|) \exp (\alpha \tau|b|) \leq \exp (\alpha(k+1) \tau|b|)
\end{aligned}
$$

as $\alpha \geq 1$. Finally, the claim follows by induction.

According to [25, Theorem 3.12], we have the following well-posedness result for (A.1).

Theorem A.4. The delay differential equation (A.1) possesses a unique strong solution $u \in L_{l o c}^{2}(-\tau, \infty ; X) \cap H_{l o c}^{1}(0, \infty ; X)$ given by

$u(t)$

$$
= \begin{cases}\varphi(t), & t \in[-\tau, 0), \\ u^{0}, & t=0, \\ \exp _{\tau}(\mathscr{B}, t-\tau) u^{0} & \\ \quad+\mathscr{B} \int_{-\tau}^{0} \exp _{\tau}(\mathscr{B}, t-2 \tau-s) u_{\tau}^{0}(s) d s & \\ \quad+\int_{0}^{t} \exp _{\tau}(\mathscr{B}, t-\tau-s) f(s) d s, & t \geq 0 .\end{cases}
$$


If $u_{\tau}^{0}$ lies in $C^{0}([-\tau, 0], X)$ and satisfies the compatibility condition $u_{\tau}^{0}(0)=u^{0}$, then the strong solution is even a classical solution; that is, $u \in C^{0}([-\tau, \infty), X) \cap C^{1}([0, \infty), X)$.

\section{Conflict of Interests}

The authors declare that there is no conflict of interests regarding the publication of this paper.

\section{Acknowledgments}

The investigations were supported by the Young Scholar Fund (Research Grant ZUK 52/2 of the Deutsche Forschungsgemeinschaft) at the University of Konstanz, Konstanz, Germany. The authors express their deep gratitude to the anonymous referee for the careful reading of the paper and the valuable comments and suggestions.

\section{References}

[1] D. E. Carlson, "Linear thermoelasticity", in Handbuch der Physik, C. Truesdell, Ed., pp. 297-346, Springer, Berlin, Germany, 1972.

[2] P. Chadwick, "Thermoelasticity. The dynamical theory," in Progress in Solid Mechanics, vol. 1, North-Holland Publishing Company, Amsterdam, The Netherlands, 1960.

[3] W. A. Day, Heat Conduction within Linear Thermoelasticity, vol. 30 of Springer Tracts in Natural Philosophy, Springer, New York, NY, USA, 1985.

[4] C. M. Dafermos, "On the existence and the asymptotic stability of solutions to the equations of linear thermoelasticity," Archive for Rational Mechanics and Analysis, vol. 29, pp. 241-271, 1968.

[5] S. W. Hansen, "Exponential energy decay in a linear thermoelastic rod," Journal of Mathematical Analysis and Applications, vol. 167, no. 2, pp. 429-442, 1992.

[6] M. Slemrod, "Global existence, uniqueness, and asymptotic stability of classical smooth solutions in one-dimensional nonlinear thermoelasticity," Archive for Rational Mechanics and Analysis, vol. 76, no. 2, pp. 97-133, 1981.

[7] C. M. Dafermos and L. Hsiao, "Development of singularities in solutions of the equations of nonlinear thermoelasticity," Quarterly of Applied Mathematics, vol. 44, no. 3, pp. 463-474, 1986.

[8] R. Racke and Y. Shibata, "Global smooth solutions and asymptotic stability in one-dimensional nonlinear thermoelasticity," Archive for Rational Mechanics and Analysis, vol. 116, no. 1, pp. 1-34, 1991.

[9] S. Jiang and R. Racke, Evolution Equations in Thermoelasticity, vol. 112 of Monographs and Surveys in Pure and Applied Mathematics, Chapman \& Hall, 2000.

[10] X. Wang and X. Xu, "Thermoelastic wave induced by pulsed laser heating," Applied Physics A: Materials Science and Processing, vol. 73, no. 1, pp. 107-114, 2001.

[11] S. A. Messaoudi and B. Said-Houari, "Exponential stability in one-dimensional non-linear thermoelasticity with second sound," Mathematical Methods in the Applied Sciences, vol. 28, no. 2, pp. 205-232, 2005.

[12] T. Irmscher, "Global existence in nonlinear hyperbolic thermoelasticity with radial symmetry," Quarterly of Applied Mathematics, vol. 69, no. 1, pp. 39-55, 2011.
[13] T. Irmscher and R. Racke, "Sharp decay rates in parabolic and hyperbolic thermoelasticity," IMA Journal of Applied Mathematics, vol. 71, no. 3, pp. 459-478, 2006.

[14] D. S. Chandrasekharaiah, "Hyperbolic thermoelasticity: a review of recent literature," Applied Mechanics Reviews, vol. 51, pp. 705-729, 1998.

[15] R. Racke, "Thermoelasticity," in Handbook of Differential Equations: Evolutionary Equations, vol. 5, pp. 315-420, Elsevier, Amsterdam, The Netherlands, 2009.

[16] D. Y. Tzou, "A unified field approach for heat conduction from macro- to micro-scales," Journal of Heat Transfer, vol. 117, no. 1, pp. 8-16, 1995.

[17] D. Y. Tzou, Macro to Micro-Scale Heat Transfer: The Lagging Behavior, Taylor \& Francis, Washington, DC, USA, 1997.

[18] M. Dreher, R. Quintanilla, and R. Racke, "Ill-posed problems in thermomechanics," Applied Mathematics Letters, vol. 22, no. 9, pp. 1374-1379, 2009.

[19] M. Fabrizio and F. Franchi, "Delayed thermal models: Stability and thermodynamics," Journal of Thermal Stresses, vol. 37, no. 2, pp. 160-173, 2014.

[20] H. M. Rodrigues, C. Ou, and J. Wu, "A partial differential equation with delayed diffusion," Dynamics of Continuous, Discrete and Impulsive Systems Series A: Mathematical Analysis, vol. 14, no. 5, pp. 731-737, 2007.

[21] D. Y. Khusainov, A. F. Ivanov, and I. V. Kovarzh, "Solution of a heat equation with delay," Nonlinear Oscillations, vol. 12, no. 2, pp. 260-282, 2009.

[22] D. Y. Khusainov, M. Pokojovy, and E. Azizbayov, "Representation of classical solutions to a linear wave equation with pure delay," Bulletin of Taras Shevchenko National University of Kyiv, Series: Cybernetics, vol. 13, no. 1, pp. 5-12, 2013.

[23] D. Y. Khusainov, M. Pokojovy, and E. Azizbayov, "Classical solvability for a linear 1D heat equation with constant delay," Zhurnal Obchyslyuval'noï ta Prykladnoï Matematyky, vol. 112, no. 2, pp. 169-195, 2013.

[24] D. Y. Khusainov, M. Pokojovy, and E. Azizbayov, "Construction of exact control for a one-dimensional heat equation with delay," Zhurnal Obchyslyuval'noï ta Prykladnoï Matematyky, vol. 115, no. 1, pp. 76-87, 2014.

[25] D. Y. Khusainov, M. Pokojovy, and R. Racke, "Strong and mild extrapolated $L^{2}$-solutions to the heat equation with constant delay," SIAM Journal on Mathematical Analysis, vol. 47, no. 1, pp. 427-454, 2013.

[26] R. A. Adams and J. J. Fournier, Sobolev Spaces, vol. 140 of Pure and Applied Mathematics (Amsterdam), Academic Press, New York, NY, USA, 2nd edition, 2003.

[27] W. Arendt, C. J. K. Batty, M. Hieber, and F. Neubrander, VectorValued Laplace Transforms and Cauchy Problems, vol. 96 of Monographs in Mathematics, Birkhäuser, Basel, Switzerland, 2001.

[28] W. H. Press and W. T. Vetterling, Numerical Recipes in Fortran 77: The Art of Scientific Computing, Cambridge University Press, New York, NY, USA, 1992. 


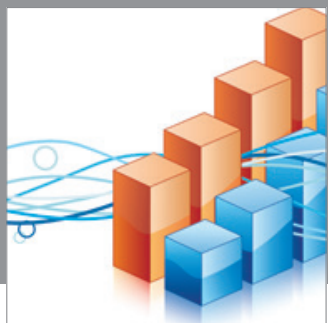

Advances in

Operations Research

mansans

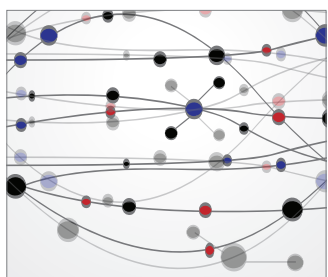

The Scientific World Journal
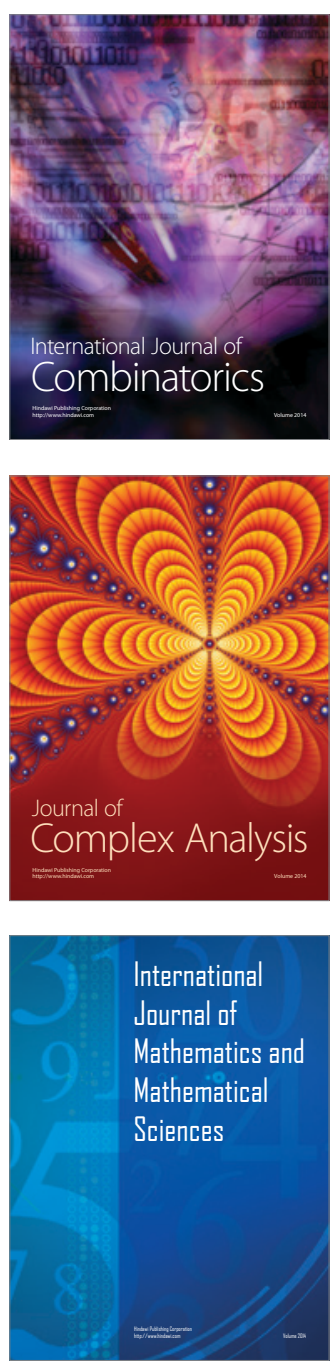
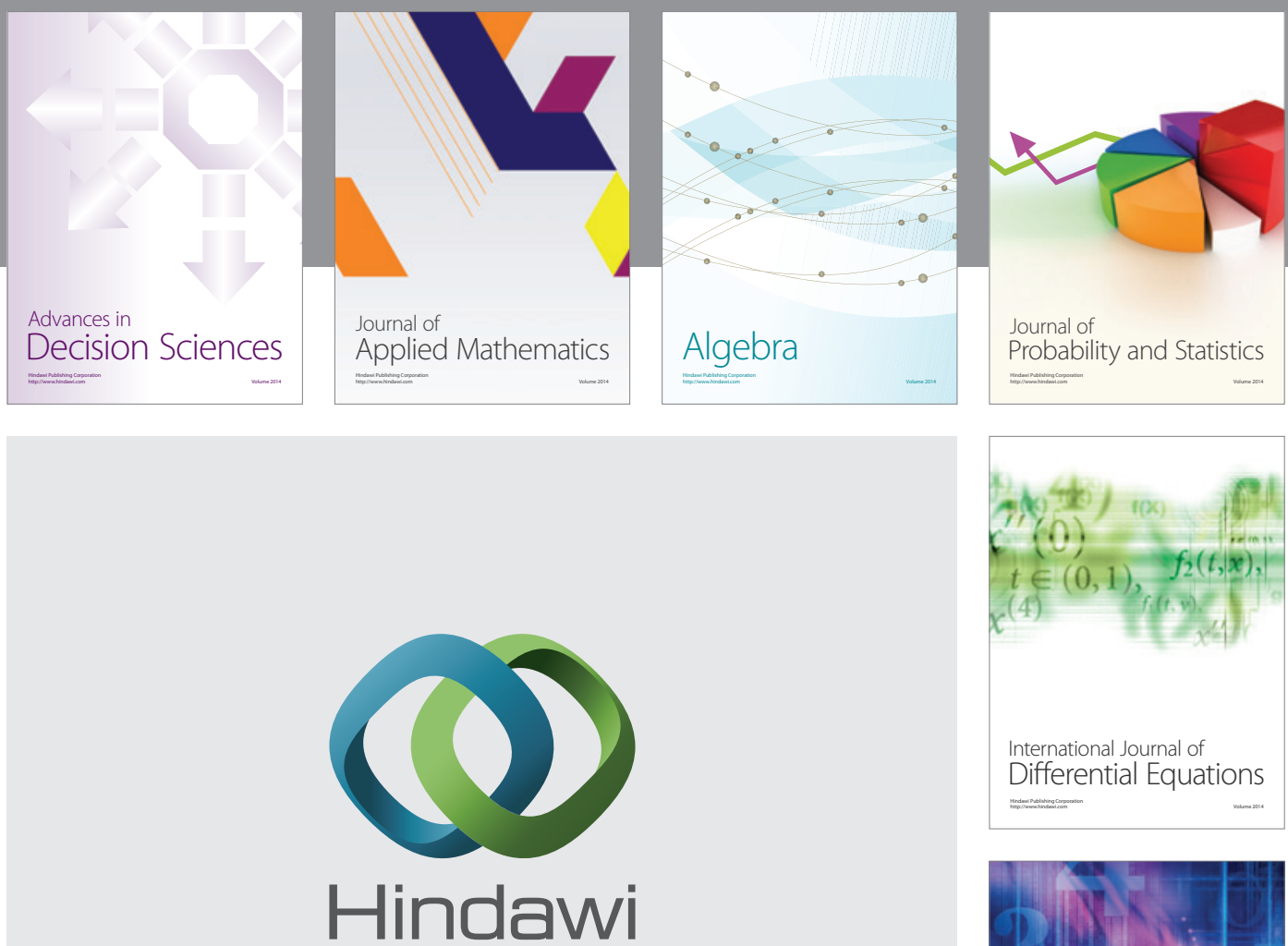

Submit your manuscripts at http://www.hindawi.com
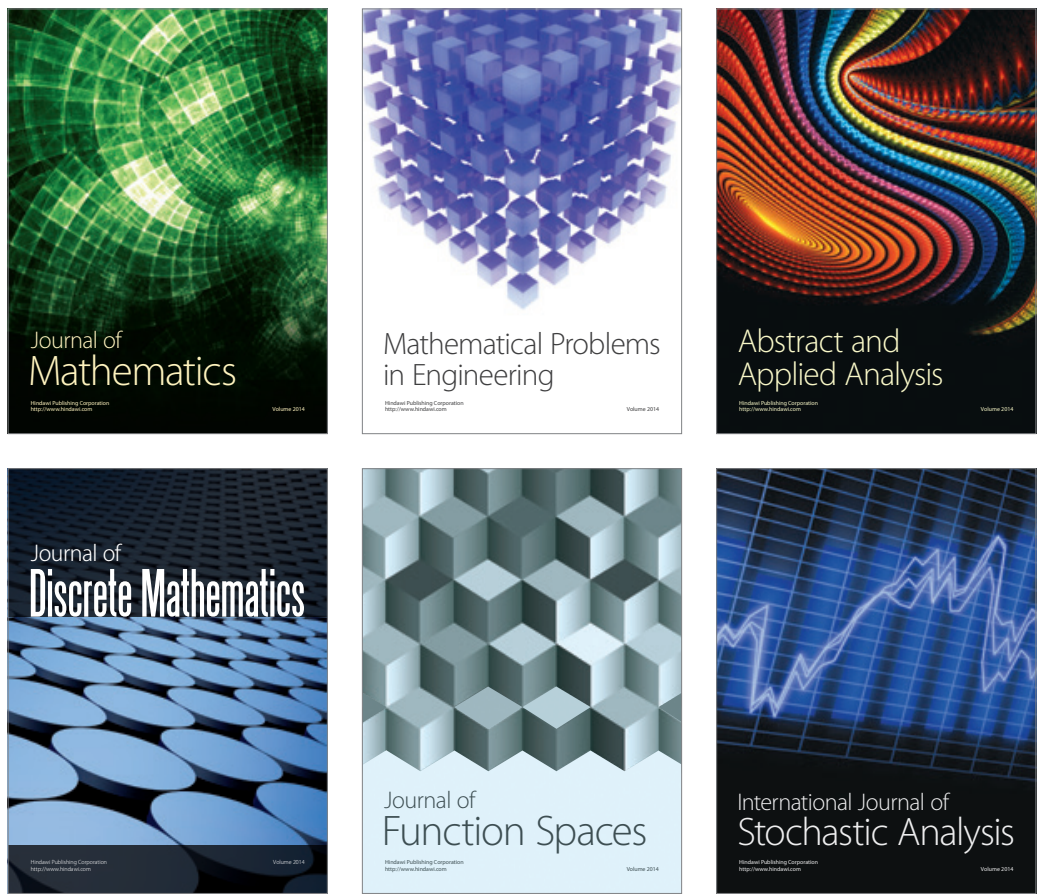

Journal of

Function Spaces

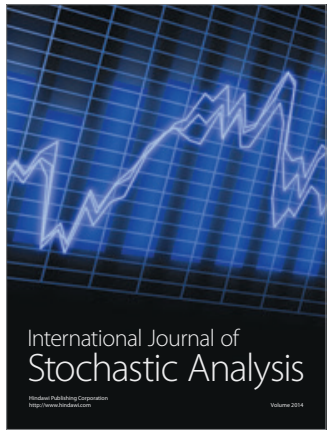

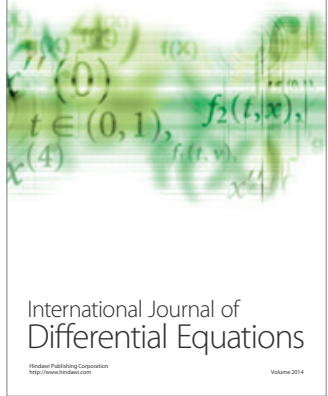
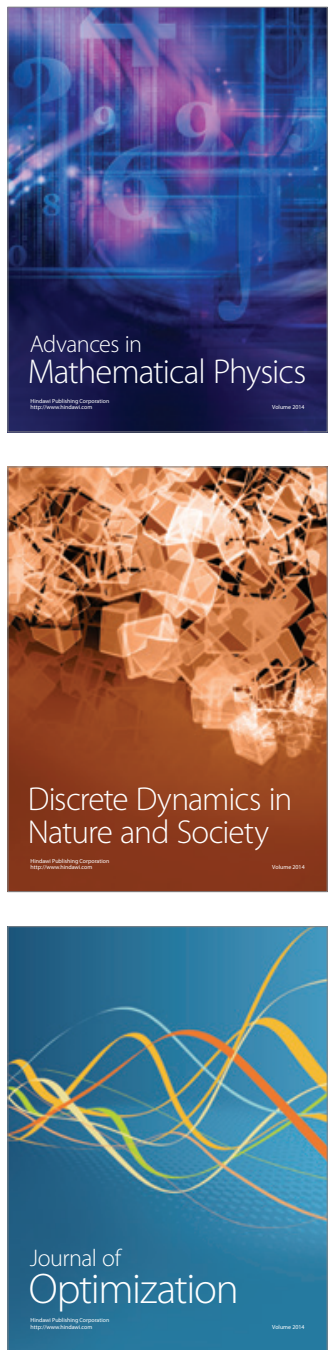